\title{
Analogy between optimal spin estimation and interferometry
}

\author{
Zdeněk Hradil and Miloslav Dušek \\ Department of Optics, Palacký University, 17. listopadu 50, 772 00 Olomouc, Czech Republic
}

(DRAFT: August 3, 2018)

\begin{abstract}
Scheme for optimal spin state estimation is considered in analogy with phase detection in interferometry. Recently reported coherent measurements yielding the average fidelity $(N+1) /(N+2)$ for $N$ particle system corresponds to the standard limit of phase resolution $1 / \sqrt{N}$. It provides the bound for incoherent measurements when each particle is detected separately and information is used optimally. For specific states, improvement up to the value $1 / N$ is possible in quantum theory. The best results are obtained combining sequentially coherent measurements on fractional groups of particles.
\end{abstract}

\section{INTRODUCTION}

Recently Peres and Wotters [1] formulated a conjecture: Coherent measurement performed on the collective system is more efficient than sequential measurements of individual particles. This idea has been further developed by Massar and Popescu [2]. They formulated this conjecture as a proposal for a "quantum game." The player has $N$ identical copies of $1 / 2$ spin particles prepared in an unknown pure state $|\psi\rangle$, and he is allowed to do any measurement. Possible results of the measurement will be denoted by an index $r$. The aim of the measurement is to determine the original state of the system. Therefore, in the next step the measured data should be attached to a state $|\psi\rangle_{r}$, which represents the players's estimation of an unknown true state. In the last stage of the game the true state is compared with its estimate and their coincidence is quantified by the so-called fidelity: $\left|\langle\psi \mid \psi\rangle_{r}\right|^{2}$. The runs are repeated many times with varying true state $|\psi\rangle$. The final score of the quantum game is given by the averaged fidelity

$$
S=\left\{\left|\langle\psi \mid \psi\rangle_{r}\right|^{2}\right\}_{a v},
$$

where averaging is carried out over the measured data and all the true states \{\}$_{a v}=\{r,|\psi\rangle\}$. Massar and Popescu proved that the maximum score is $(N+1) /(N+$ $2)$. This value cannot be reached by measurements acting on isolated particles. Derka, Bužek and Ekert 3] showed that this score can be obtained by coherent measurement described by a finite-dimensional probability operator valued measure.

The aim of this contribution is to address the relation between recently optimized measurement, repeated measurement on the Stern-Gerlach apparatus and interferometry. Particularly, it will be demonstrated that the above mentioned score represents the ultimate limitation for sequential measurements performed on each particle separately for any quantum state. This corresponds to the standard resolution $1 / \sqrt{N}$ currently reached in interferometry. However, this regime does not represent the ultimate strategy. In analogy with quantum interferometry performance may achieve the resolution up to $1 / N$ provided that spin orientation is properly coded into a quantum state of $N$ particles. In the particular case addressed in this contribution the optimal strategy corresponds to sequentially performed coherent measurements. This explicit example demonstrates the complexity of optimal measurement, which can combine advantages of both the coherent and sequential measurements with groups of particles.

\section{ADAPTIVE STERN-GERLACH SPIN DETECTION}

Assume a standard measurement on an ideal SternGerlach apparatus. A sample particle is prepared in the pure spin state

$$
|\mathbf{n}\rangle\langle\mathbf{n}|=\frac{1}{2}[1+(\mathbf{n} \sigma)]
$$

where $\mathbf{n}$ represents the unity vector on the Poincare sphere, $(\mathbf{n} \sigma)$ being its scalar product with vector of Pauli matrices. The impinging particles will be deviated up or down. In the long run of repetitions the relative frequencies will approach the prediction of quantum theory. Representing the setting of the Stern-Gerlach apparatus by the unity vector $\mathbf{m}$, the probabilities of detection "up" $(+)$ and "down" $(-)$ read

$$
p_{ \pm}=\frac{1}{2}[1 \pm(\mathbf{m n})]
$$

What is the best possible but still feasible result, which would predict the spin orientation with the highest accuracy? The most accurate state estimation may be done if all tested particles were registered in the same output channel of the SG apparatus. In such a case the best estimation of the spin corresponds to the orientation of the SG apparatus. Of course, it does not mean that the estimated direction will fit the spin orientation exactly. Deviations are distributed according to the posterior distribution conditioned by the detected data. This can be 
handled analogously to the phase estimation 画. The deviations between the estimated and the true directions are given by the Bayes theorem as the posterior probability density

$$
P(\mathbf{n})=\frac{N+1}{4 \pi} \cos ^{2 N}(\theta / 2)
$$

over the Poincaré sphere. The vector $\mathbf{n}$ is parametrized by the Euler angles $\theta, \phi$ in the coordinate system where the direction of $z$-axis coincide always with the estimated direction (i.e. with the orientation of SG apparatus $\mathbf{m}$ ). Notice that this is in accordance with the rules of the quantum game as defined in [2]. The result of the measurement is always a specific direction, namely the setting of SG apparatus. In this case the score reads

$$
S=\int P(\mathbf{n}) d^{2} \Omega_{\mathbf{n}} \cos ^{2}(\theta / 2)=\frac{N+1}{N+2} .
$$

This is the upper bound of sequential measurements on single particles with felicitously rotated SG apparatus. This is obviously an ultimate limitation since such results are possible and none measurement performed sequentially on single particles can yield better spin prediction. However, on the contrary to the coherent measurements, this resolution cannot be really achieved, but it may be approximated with an arbitrary accuracy for $N$ large enough.

The possible realization may be suggested as an adaptive scheme, where the orientation of SG device depends on the previous results. The aim of the scheme is to find such an orientation, where almost all the particles are counted on the same port. This is obviously always a little worse than ideal case, since some portion of counted particles must always be used for corrections of the SGdevice orientation and are therefore "lost". Differences between ideal and realistic scheme seem to be negligible as demonstrated in Fig. 11. An adaptive measurement is simulated here. The procedure starts with projecting a single particle into the three orthogonal randomly chosen directions. The choice of the orientation of the subsequent SG measurement represents its own interesting optimization problem. Obviously, for getting the best score, it seems to be advantageous to project the particles into the most probable orientation. However, this will not reveal new corrections to the orientation of SG apparatus well. On the other hand, the particles may be counted with the SG device oriented perpendicularly to the most probable orientation. In this case the spreading of the data will be obviously broader than in the former case, but such measurement will be more sensitive to the deviation from the true direction. In the simulation scheme, the latter approach has been used and algorithm for synthesis of incompatible spin projections has been used [5]. The treatment is still not optimal, however, as will be seen in the next paragraph, the differences seem to be unimportant.

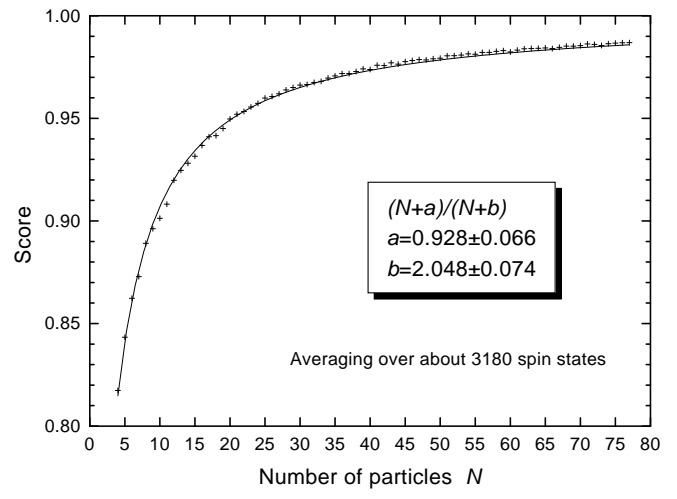

FIG. 1. Numerical simulation of one-particle adaptive measurement.

\section{ANALOGY BETWEEN SPIN ESTIMATION AND INTERFEROMETRY}

There is a clear analogy between spin measurement and phase interferometry. As the proper resolution measure, the dispersion of phase may be defined as [6,7]

$$
D^{2}=1-\{\cos \theta\}_{a v}^{2} .
$$

Usually, the averaging is done over the data only. Provided that the width of phase distribution is small, dispersion tends to the standard variance of phase. Using the definition of the score

$$
S=\frac{1}{2}+\frac{1}{2}\{\cos \theta\}_{a v}
$$

both the measures fulfil the relation

$$
S(S+1)=\frac{D^{2}}{4} .
$$

Consequently, the value $S=(N+1) /(N+2)$ is nothing else as dispersion (phase variance) $D \approx 2 / \sqrt{N}$. This is the so-called standard limit of phase resolution [8,9]. Any standard measurement is scaled in this way and all the measurements differ by some multiplicative factors only. Loosely speaking, all the classical strategies are essentially equivalent from this viewpoint. This is why it has perhaps little sense to optimize further the adaptive scheme. For example, provided that one will use the most straightforward method of spin estimation based on the measurement of $x, y$, and $z$ components of the spin on the Poincaré sphere always with $N / 3$ particles, the resulting score may be evaluated asymptotically as $S \approx 1-114 /(100 N)$. The difference between optimal coherent and realistic sequential measurements is assumed sometimes to be significant for small number of particles. However, in this case all the predictions are rather uncertain. In the case of phase detection it has little sense to compare two phase distributions, whose widths are comparable to $2 \pi$ window. Then the phase knowledge is almost equally bad. Conventional resolution measures 
possess good meaning only when the effective width is substantially less than the width of the interval.

More profound analogy between the spin measurement and interferometry follows from the common nature of the $\mathrm{SU}(2)$ symmetry [8]. As well known, the resolution up to the order $1 / N$ may be achieved in interferometry. In this case, however, it is recommended to modify slightly the proposed quantum game. Suppose that somebody wants to communicate an orientation of the axis in 3D space. Just an axis, not the direction of an "arrow". For this purpose $N 1 / 2$-spin particles are available and any measurement on these particles is allowed. Instead of $N$ identical copies, one may consider a general quantum state spanned by $N$ particles, in which the unknown orientation is coded. As the result of the measurement the unknown axis should be find. The score is defined in the same manner as before. The question are: "How to encode information on axis orientation into a quantum state of the particles? What measurement should be done in order to obtain the best score?"

\section{SUPERRESOLUTION IN INTERFEROMETRY AND SPIN ESTIMATION}

Let us review briefly the description of $\mathrm{SU}(2)$ interferometers. The transformation of an internal state is given by an unitary transformation of an input state

$$
\begin{array}{r}
\hat{U}(\theta, \varphi)=\exp \left[-i \theta \hat{J}_{2}(\varphi)\right] \equiv \\
\exp \left(i \varphi \hat{J}_{3}\right) \exp \left(-i \theta \hat{J}_{2}\right) \exp \left(-i \varphi \hat{J}_{3}\right)
\end{array}
$$

where $\hat{J}_{1}, \hat{J}_{2}, \hat{J}_{3}$ corresponds to generators of $\mathrm{SU}(2)$ group [8,9]. The transformation is given by the unity vector $\mathbf{n}=(\cos \varphi \sin \theta, \sin \varphi \sin \theta, \cos \theta)$. An input state $\mid$ in $\rangle$ may be any $N$ particle state. The measurement may be represented by projectors into an output state $\mid$ out $\rangle$. The posterior phase distribution corresponds to the scattering amplitude

$$
P(\theta, \varphi) \approx \mid\left.\langle\text { in }|\hat{U}(\theta, \varphi)| \text { out }\rangle\right|^{2} .
$$

This scheme encompasses the Massar, Popescu quantum game as a special case for the choice of the input state $\mid$ in $\rangle=|N, N\rangle$. Score depends on the accuracy of detection of $\theta$ variable. As well known, the highest accuracy is achieved when the phase shift near the zero value is detected. This corresponds to the detection of the same quantum state on the output $\mid$ out $\rangle=|N, N\rangle$. The particles feeding a single input port of an interferometer appears again on the corresponding output port. Obviously, the interpretation does not change provided that particles will enter and leave the interferometer sequentially. This is the consequence of the famous Dirac statement that "each particle interferes with itself." 10 Ultimate score $(N+1) /(N+2)$ is relevant just to this regime. Considerations related to adaptive $\mathrm{SG}$ detection are obviously related also to this situation.
In the following, for given input state and for given setting of the parameters of the interferometer, only the most favourable but still feasible output will be assumed. Such felicitous results provide an upper bound conditioned by the realistic measurements. Obviously, no other measurement of the given type, can provide better prediction.

As well known in interferometry 8,9,11, , better resolution may be obtained provided that both the input ports of an interferometer are fed simultaneously by an equal number of $r$ particles. In the case when the same output state appears on the output, the phase shift prediction will be the sharpest. This corresponds to the input and output states $\mid$ in $\rangle=|2 r, 0\rangle$ and $\mid$ out $\rangle=|2 r, 0\rangle$. The scattering amplitude then depends only on the variable $\theta$ as

$$
P(\theta) \propto\left[P_{r}^{0}(\cos \theta)\right]^{2}
$$

The Legendre function $P_{r}^{0}(\cos \theta)$ may be approximated by Bessel function $J_{0}(r \theta)$ for large index $r$. Consequently, the probability distribution is not integrable for $r \rightarrow \infty$ and must be therefore treated more carefully [12]. Particularly, it is therefore not the best strategy to use all the energy of $N$ states in the single coherent measurement. The particles should be divided into several groups and the measurement should be sequentially repeated several times. The accumulation of information is expressed mathematically by the multiplication of corresponding distribution functions (11). This tends to narrowing of the posterior probability distribution. The optimal regime for the $\theta$ detection has been roughly estimated in Ref. [12]. Optimal number of repetitions of the coherent measurement was expected to be approximately $n \approx 4$.

Let us interpret this interferometric measurement in terms of the spin estimation. The significant difference between spin $1 / 2$ particles and photons is connected with their fermionic and bosonic nature. The input wave function constructed from fermions must be "artificially" symmetrized with respect to all the distinguishable particles. The input state $|N, 0\rangle$ is a superposition involving all the combinations of $N / 2$ particles with the spin up and $N / 2$ particles with the spin down. This $N$ particle state is entangled.

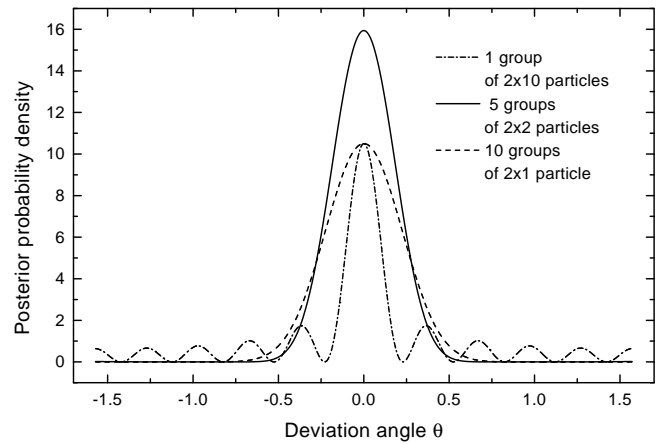

FIG. 2. The posterior probability distribution provided that the exactly same number of spins "up" and "down" has been detected. The total number of particles is 20 . 
Let us analyse in detail the the score for such states. As before the most favourable but still physically feasible situation will be interpreted as an ultimate bound of the state detection. Due to the symmetry of the problem, the best resolution may be inferred provided that the state $|N, 0\rangle$ feeds the $\mathrm{SG}$ apparatus. The results of such measurement will be deterministic in the case when the apparatus is oriented along the spin state of each particle. In this case a half of particles will be detected with the spin "up" and a half of particles with the spin "down". Assume now that this really happened. What can be inferred from this event? Obviously, this might occure also in the case when the setting of SG differs from the right spin orientation by an angle $\theta$. The probability that this happens is proportional to the scattering amplitude (11). The probability is sketched in Fig. 2-as can be seen it shows oscillations. The domain is restricted to the values $(0, \pi / 2)$ since the method just finds the axis but not its vector orientation. The score is plotted in Fig. 3. As shown it does not improve with increasing number of particles. It is caused by the heavy tails of the posterior distribution (Fig. 2), which does not contain the dominant amount of the probability in the central peak. Nevertheless, there is a way how to suppress this undesired behaviour. Provided that the measurement is repeated, the corresponding posterior distribution will be given by the product of partial results. Again, the most favourable situation for spin estimation is characterized by the repetition of the same "optimal" result. This is again feasible, provided that the true spin orientation and projection do not differ significantly. The resulting score in dependence on the total number of particles is sketched in Fig. 3. Notice, that SG measurement must be done with $N / 2$ particle state in this case, since the measurement is repeated twice. This procedure may be further generalized including an arbitrary number of repetitions. As shown in Fig 3, the score increases up to the 5 repetitions and then starts to decrease. This seems to be in good agreement with the rough asymptotical analysis presented in Ref. [12], where the optimal repetition rate has been found as 4 .

This result provides an ultimate bound in the following sense: If the given input state is measured with the help of SG projections, the score cannot be better than this ultimate value. The question whether this value may be attained is not answered here. Intuitively, in the case of large number of particles it might be well approximated by an adaptive scheme as in previous case of standard resolution. However, the adaptive scheme cannot be applied to the first measurement. The results may therefore appear as overestimated in this case. This explains why the analysis applied here provides the score $S \approx 0.875$ for the state $|\uparrow, \downarrow\rangle$, whereas the result of Gisin and Popescu [15] gives the value 0.789 . In the asymptotical limit, the dispersion is approximately given by the relation

$$
D \approx \frac{\sqrt{4 n}}{N}=\frac{2 \sqrt{5}}{N} .
$$

The optimal score reads asymptotically

$$
S \approx 1-\frac{5}{N^{2}}
$$

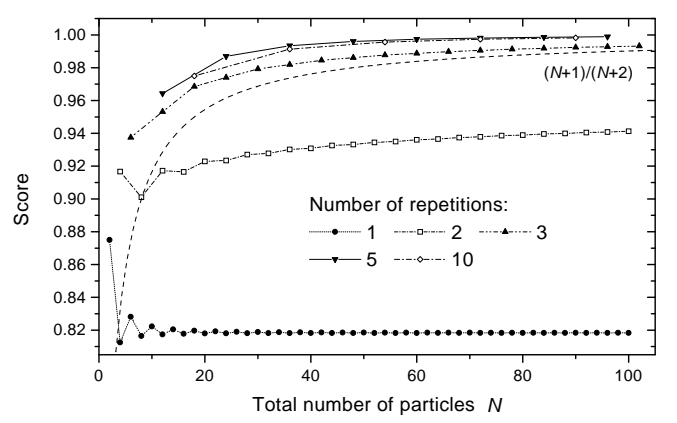

FIG. 3. Ultimate values of score for several repetitions of coherent measurements on groups of particles.

One may ask whether the required states containing half particles with spins "up" and half with spins "down" (in an arbitrary direction) can be prepared from a set of particles with all spins "up", i.e. if it is possible to "turn" arbitrary quantum state into a state orthogonal to it. The general answer is no - the linearity of quantum mechanics does not allow this. Particularly in case of $1 / 2$-spin particles, if one is able to do: $|\uparrow\rangle \rightarrow|\downarrow\rangle$ and $|\downarrow\rangle \rightarrow e^{i \varphi}|\uparrow\rangle$ then for an arbitrary state it follows from linearity of quantum evolution that

$$
|\Psi\rangle=(\alpha|\uparrow\rangle+\beta|\downarrow\rangle) \rightarrow\left(\alpha|\downarrow\rangle+e^{i \phi} \beta|\uparrow\rangle\right) .
$$

The last state is orthogonal to $|\Psi\rangle$ if and only if $\arg (\alpha)+$ $\pi=\arg (\beta)+\phi$. Thus in special situations (corresponding to "real" subspaces) the mentioned transformation is possible (e.g. when spin projections lay in a given plane, also for linear polarizations of photon or for interferometry with fixed splitting ratios and varied a phase difference only). But an arbitrary spin cannot be turned to the perfectly opposite one.

\section{CONCLUSIONS}

The profound analogy between interferometry and spin estimation has been addressed. The performance of both the schemes discussed has been conditioned by the realistic measurements only. As demonstrated, the recently reported optimal spin estimation corresponds to the standard quantum limit characterized by the resolution $1 / \sqrt{N}$. It may be achieved by coherent measurements and well approximated by sequential ones. Beyond this regime, the quantum theory admits the resolution up to $1 / N$. Then, however, quantum interferences must be employed. In the case of a thought experiment with an ensemble of spin $1 / 2$ particles it requires an entangled input state of $N$ particles. Besides this, optimal SG detection must combine advantages of both the coherent 
and sequential measurements. This example illustrates complexity of the optimal treatment in estimation problems.

\section{ACKNOWLEDGMENTS}

Discussions with V. Bužek and D. Terno are gratefully acknowledged. This work has been supported by the grant VS 96028 and by the research project "Wave and particle optics" of the Czech Ministry of Education and by the grant No. 19982003012 of the Czech National Security Authority.

[1] A. Peres, W.K. Wootters, Phys. Rev. Lett. 66, 1119 (1991).

[2] S. Massar, S. Popescu, Phys. Rev. Lett. 74, 1259 (1995).

[3] R. Derka, V. Bužek, A.K. Ekert, Phys. Rev. Lett. 80 1571 (1998).

[4] Z. Hradil, R. Myška, J. Peřina, M. Zawisky, Y. Hasegawa and H. Rauch, Phys. Rev. Lett. 760, 4295 (1996); M. Zawisky, Y. Hasegawa, H. Rauch, Z. Hradil, R. Myška, J. Peřina, J. Phys. A : Math. Gen. 31, 551 (1998).

[5] Z. Hradil. J. Summhammer, G. Badurek, H. Rauch, "Reconstruction of spin states" in preparation.

[6] R.C. Rao, Linear Statistical Inference and Its Applications, (New York, Wiley, 1973), p.175.

[7] Z. Hradil, Quantum Opt. 4, 93 (1992).

[8] B. Yurke, S.L. McCall, J.R. Klauder, Phys. Rev. A 33, 4033 (1986).

[9] B.C. Sanders, G.J. Milburn, Phys. Rev. Lett. 75, 2944 (1995)

[10] P.A.M. Dirac, The principles of quantum mechanics, (Claredon Press, Oxford, 1947).

[11] M.J. Holland and K. Burnett, Phys. Rev. Lett. 71, 1355 (1993).

[12] Z. Hradil. R. Myška, Acta phys. slov. 46, 405 (1996).

[13] Z. Hradil, Phys. Rev. A 55, R1561 (1997).

[14] Z. Hradil, J. Summhammer, H. Rauch, "Quantum tomography as normalization of incompatible observations", submitted.

[15] N. Gisin, S. Popescu, e-print quant-ph/9901072. 